\title{
ACQUIRED HEMOPHILIA A AS A MANIFESTATION OF SJÖGREN'S SYNDROME: RITUXIMAB TREATMENT EXPERIENCE
}

Raíssa Dudienas Domingues Pereira, ${ }^{1, *}$, Vinicius Verlangieri Soubihe ${ }^{1}$, Caio Rustichelli Cardoso ${ }^{1}$, Diego de Paula Ferreira Nunes ${ }^{1}$, Margareth Castro Ozelo ${ }^{1}$, Alisson Pugliesi ${ }^{1}$, Zoraida Sachetto ${ }^{1}$

1.Universidade Estadual de Campinas, Campinas (SP), Brazil.

*Corresponding author: ra_dudienas@hotmail.com

\section{BACKGROUND}

Acquired hemophilia (AH) is a rare bleeding disorder, caused by antibodies against a clotting factor, usually antifactor VIII. In some cases, antifactor VIII can occur in patients with no relevant comorbidities, but in 40-50\% it can be associated with postpartum period, cancer or autoimmune diseases, such as Sjögren's syndrome (SS). The treatment is based on hemostatic therapy during bleeding and immunosuppressive therapies to eradicate the inhibitor, usually with corticosteroids in combination with cytotoxic agents, such as cyclophosphamide. We bring here a case report of type A AH as a manifestation of SS, with intolerance to treatment with oral cyclophosphamide but response to rituximab.

\section{CASE REPORT}

Female, 57 years old, in follow-up with rheumatology due to SS, using intravenous cyclophosphamide due to vasculitis, with multiple mononeuritis and skin lesions (purpura and livedo). In 2020, after hemorrhagic manifestations, such as bleeding from the gastrointestinal tract and muscle hematoma after peripheral venipuncture, laboratory tests revealed changes in the coagulogram, with increased activated partial thromboplastin time. Due to this change, the dosage of coagulation factor VIII was continued, which shown to be reduced, with the presence of factor VIII inhibitor, and type A AH was diagnosed. Due to severe bleeding, an infusion of activated prothrombin complex was indicated and, due to hemophilia, immunosuppression with oral cyclophosphamide was initiated. However, about 15 days after starting cyclophosphamide, the patient evolved with bicytopenia (hemoglobin of 5.4 and lymphopenia of 670), a condition compatible with myelotoxicity by the drug and, therefore, opted to discontinue the medication, with subsequent recovery of hematimetric levels. She evolved with fever, lymphadenopathy and relapse of hemophilia. As a patient developed hemophilia during the use of intravenous cyclophosphamide and had important side effects with the use of oral cyclophosphamide, we opted for treatment with rituximab, in the regimen of two doses of $1 \mathrm{~g}$ each, spaced for 15 days. After the first course of rituximab, the patient presented significant clinical and laboratory improvement, with no new skin lesions and AH control. The patient remains on rituximab, with no relapses of the disease since starting treatment, about a year ago.

\section{CONCLUSION}

After failure of other immunosuppressive agents, rituximab is a drug that can be considered in the treatment of severe and refractory SS. In this case, we have a satisfactory effect of its use for this serious and rare manifestation of SS.

\section{KEYWORDS}

Sjögren's syndrome, Acquired hemophilia, Rituximab. 